\title{
DESCENDING CHAIN CONDITIONS FOR GRADED RINGS
}

\author{
M. SAORÍN
}

(Communicated by Maurice Auslander)

\begin{abstract}
It is proved that if $R$ is a perfect (resp. Artinian) strongly graded ring whose ground subring is, modulo its Jacobson radical, a finite direct product of finite-dimensional simple algebras over (nondenumerable) algebraically closed fields, then the grading group cannot contain an infinite abelian subgroup (resp. must be finite). These results extend those of A. Reid and D. S. Passman on twisted group algebras.
\end{abstract}

In the early seventies Renault [14] and Woods [15] proved that a group ring $A G$ is perfect (resp. Artinian) if and only if $A$ is perfect (resp. Artinian) and $G$ is finite. There is no corresponding result for twisted group algebras, as Passman's surprising example (see, e.g., [11, Proposition 4.3]) of a field that is a twisted group algebra of an infinite group shows. Notwithstanding, Passman also showed that if $K$ is a nondenumerable algebraically closed field, then $G$ must be finite in case $K^{t} G$ is Artinian. Later on, Reid [13, Theorem 2.6] skipped the hypothesis of nondenumerability and proved that the group $G$ could not contain an infinite abelian subgroup, thus eliminating the possibility of $G$ being, for instance, infinite abelian or infinite locally finite. On the order hand, Keok Park proved in [8, Theorems 3.3 and 3.7] that the skew group ring versions of the Renault-Woods theorems also held.

Putting together all the above facts encouraged us to study what happens in the more general situation in which $R=\bigoplus_{\sigma \in G} R_{\sigma}$ is just a strongly graded ring, with some restrictions of algebraic closure on the underlying fields that eventually appear. In one direction, namely, if $R_{1}$ is perfect (resp. Artinian) and $G$ is finite then $R$ is perfect (resp. Artinian), the result has been established in the perfect case by Jensen and Jondrup [6, Theorem 4.1], even without the strong gradation hypothesis and is easily deducible by using the duality theory of Cohen and Montgomery [4] in the strongly graded Artinian case. Our goal in this paper is to check the other direction, and it turns out that the results of Reid and Passman can be carried to our rather more general situation (Theorems 5 and 12).

Throughout, all rings are associative with 1 . If $R$ is a $G$-graded ring, $R_{\sigma}$ will be the $\sigma$ th homogeneous component of the ring, for every $\sigma \in G$, and if

Received by the editors November 16, 1990.

1991 Mathematics Subject Classification. Primary 16A03; Secondary 16A35, 16A51.

This work was finished while the author was visiting the University of Iowa, Iowa City, supported by the DGICYT. It was presented at the AMS meeting in San Francisco on January 17, 1991. 
$1 \in G$ is the netural element of the group, $R_{1}$ will be sometimes referred to as the ground ring of the gradation. If $R_{\sigma} R_{\tau}=R_{\sigma \tau}$ for all $\sigma, \tau \in G$, the ring is called strongly graded. If $H$ is a subgroup of $G$ then $R^{(H)}$ will denote the $H$-graded subring of $R$ given by $R^{(H)}=\bigoplus_{h \in H} R_{h}$. If moreover $H$ is normal in $G$, then $R$ can be given a $G / H$-gradation with ground ring $R^{(H)}$ by defining $R_{x}=\bigoplus_{\sigma \in x} R_{\sigma}$ for any left coset $x \in G / H$. The graded Jacobson radical of $R$ will be denoted by $J^{g}(R)$. For all terminology about graded rings included in the paper, we refer the reader to [10].

If $R$ is $G$-graded, then we have an exact sequence of groups $1 \rightarrow U\left(R_{1}\right) \hookrightarrow$ $h U(R) \stackrel{d}{\rightarrow} G$, where $U\left(R_{1}\right)$ is the group of units of $R_{1}, h U(R)$ is the group of homogeneous units of $R$, and $d(x)$ is the degree of $x$ for any $x \in h U(R)$; i.e., $d(x)$ is the unique $\sigma \in G$ such that $x \in R_{\sigma}$. When $d$ is also surjective, the graded ring is said to be a $G$-crossed product with ground ring $R_{1}$. In that situation, if we choose a section $u: G \rightarrow h U(R)\left(\sigma \rightarrow u_{\sigma}\right)$ for $d$, i.e., a map such that $d \circ u$ is the identity map of $G$, satisfying $u_{1}=1$, then $\left\{u_{\sigma} / \sigma \in G\right\}$ is a basis of $R$ as both a left and right $R_{1}$-module, conjugation by $u_{\sigma}$ yields an automorphism of $R_{1}$, and $u_{\sigma} u_{\tau} u_{\sigma \tau}^{-1} \in \operatorname{Ker} d=U\left(R_{1}\right)$. In such a way we get maps $\varphi: G \rightarrow \operatorname{Aut}\left(R_{1}\right)$ (called the action of $G$ on $R_{1}$ ), given by $\varphi(\sigma)=$ conjugation by $u_{\sigma}$, and $t: G \times G \rightarrow U\left(R_{1}\right)$ (called the twisting), given by $t(\sigma, \tau)=u_{\sigma} u_{\tau} u_{\sigma \tau}^{-1}$. Both maps are related by the following formulas:

(C1) $\left(a^{\tau}\right)^{\sigma}=t(\sigma, \tau) a^{\sigma \tau} t(\sigma, \tau)^{-1}$,

(C2) $t(\sigma, \tau) t(\sigma \tau, \vartheta)=t(\tau, \vartheta)^{\sigma} t(\sigma, \tau \vartheta)$

(C3) $t(\sigma, 1)=t(1, \sigma)=1$,

for every $a \in R_{1}$ and $\sigma, \tau, \vartheta \in G$, where $a^{g}=\varphi(g)(a)$ for each $a \in R_{1}$ and $g \in G$. The procedure is reversible and if we have a group $G$ and a ring $A$, with maps $\varphi: G \rightarrow \operatorname{Aut}(A)$ and $t: G \times G \rightarrow U(A)$ satisfying $\mathrm{C} 1-\mathrm{C} 3$, then we can form a crossed product by taking the free left $A$-module $A * G$ with basis a copy $\{\bar{\sigma} / \sigma \in G\}$ of $G,(a * G)_{\sigma}=A \bar{\sigma}$ and extending by linearity the product $(a \bar{\sigma})(b \bar{\tau})=a b^{\sigma} t(\sigma, \tau) \overline{\sigma \tau}$.

We shall write $R=R_{1} * G$ to indicate that $R$ is a $G$-crossed product with ground ring $R_{1}$. If $H$ is a subgroup of $G$ then $R^{(H)}=R_{1} * H$ and, in the particular case when $H$ is normal, the $G / H$-gradation of $R$ with ground ring $R^{(H)}$ gives an exact sequence of groups: $1 \rightarrow U\left(R^{(H)}\right) \hookrightarrow \hat{h} U(R) \stackrel{\bar{d}}{\rightarrow} G / H \rightarrow 1$, where $\hat{h} U(R)$ is the group of homogeneous units of $R$ with respect to that $G / H$-gradation. A section $\bar{u}: G / H \rightarrow \hat{h} U(R)$ can be given as follows: we fix a set of representatives $\left\{g_{i} / i \in I\right\}$ containing 1 of the left cosets modulo $H$ and define $\bar{u}_{g_{i} H}=u_{g_{i}}$, where $u$ is a previously fixed section of $d$ in the $G$-crossed product structure of $R$. In this way, if $\left\{\bar{\sigma}=u_{\sigma} / \sigma \in G\right\}$ is the left $R_{1}$-basis of $R$, then the action of $g_{i} H \in G / H$ on $R^{(H)}=R_{1} * H$ is just conjugation by $u_{g_{i}}=\bar{g}_{i}$. A particular case of this situation occurs when $H=G_{\mathrm{inn}}$ is the subgroup of $G$ consisting of those $\sigma \in G$ whose action on $R_{1}$ is inner, i.e., there exists a $w \in U\left(R_{1}\right)$ such that $a^{\sigma}=w a w^{-1}$, for any $a \in R_{1}$.

For all terminology about crossed products included in the paper, the reader is referred to [7] and [12]. All the ring-theoretic tools used can be found in [1].

In order to prove the first result, we need two lemmas. 
Lemma 1. If $R$ is a G-graded left perfect (resp. Artinian) ring, then $R^{(H)}$ is also a left perfect (resp. Artinian) ring for any subgroup $H$ of $G$.

Proof. It is clear that $R^{(H)}$ is a direct summand of $R$ both as a left and right $R^{(H)}$-module. Then Proposition 2.1 of [8] gives the result when we use the characterizations by means of descending chain conditions of perfect and Artinian ring.

Lemma 2. Let $R$ be a $G$-graded ring and $\tau \in G$. The following assertions are equivalent:

(i) $J^{g}(R)_{\tau}=R_{\tau}$,

(ii) $R_{\tau} R_{\tau^{-1}} \subseteq J\left(R_{1}\right)$,

(iii) $J^{g}(R)_{\tau^{-1}}=R_{\tau^{-1}}$.

Proof. We show the equivalence (i) $\Leftrightarrow$ (ii) and (ii) $\Leftrightarrow$ (iii) will follow by symmetry. We use the easily deducible fact that $x \in R_{\tau}$ is an element of $J^{g}(R)$ if and only if $1-x a \in U\left(R_{1}\right)$ for each $a \in R_{\tau^{-1}}$ (resp. $1-a x \in U\left(R_{1}\right)$ for each $\left.a \in R_{\tau^{-1}}\right)$. With that in mind, $J^{g}(R)_{\tau}=R_{\tau}$ iff $1-R_{\tau} R_{\tau^{-1}}=\left\{1-x y / x \in R_{\tau}\right.$, $\left.y \in R_{\tau^{-1}}\right\} \subseteq U\left(R_{1}\right)$. But since $x R_{\tau^{-1}}$ is a right ideal of $R_{1}$, the latter condition is equivalent to $x R_{\tau^{-1}} \subseteq J\left(R_{1}\right)$ for any $x \in R_{\tau}$, i.e., $R_{\tau} R_{\tau^{-1}} \subseteq J\left(R_{1}\right)$.

And we can now give our first result.

Proposition 3. Let $R$ be a left perfect $G$-graded ring such that for any cyclic subgroup $H$ of $G,\left\{\sigma \in H / R_{\sigma} R_{\sigma^{-1}} \subseteq J\left(R_{1}\right)\right\}$ is finite. Then $G$ is a periodic group.

Proof. Suppose $G$ is not periodic and $x \in G$ is an element of infinite order. Then we take $H=\langle x\rangle$ and the graded subring $R^{(H)}$. By Lemma $1 R^{(H)}$ is left perfect. But $R^{(H)}$ may be viewed as a $\mathbb{Z}$-graded ring and hence $J\left(R^{(H)}\right) \subseteq$ $J^{g}\left(R^{(H)}\right)$ (see, e.g., [10, Remark I.7.16]). Now $R^{(H)} / J^{g}\left(R^{(H)}\right)$ is a factor ring of the semisimple Artinian ring $R^{(H)} / J\left(R^{(H)}\right)$ so that it must be itself semisimple Artinian. By [3, Proposition 8], $R^{(H)} / J^{g}\left(R^{(H)}\right)$ must be finitely graded and hence $\left\{\tau \in H /\left(R^{(H)} / J^{g}\left(R^{(H)}\right)\right)_{\tau}=0\right\}=\left\{\tau \in H /\left(R^{(H)}\right)_{\tau}=J^{g}\left(R^{(H)}\right)_{\tau}\right\}$ is cofinite in $H$. By Lemma 2, we get that $\left\{\tau \in H / R_{\tau} R_{\tau^{-1}} \subseteq J\left(R_{1}\right)\right\}$ is cofinite in $H$ and thus infinite, contradiciting the assumption.

Example 4. Let $S$ be a primitive ring that is not simple (e.g., the endomorphism ring of an infinite-dimensional vector space). For any choice of nonzero proper ideal $I$ of $S$, the $\mathbb{Z}$-graded subring of the Laurent polynomial ring $S\left[X, X^{-1}\right]$ given by

$$
R=\cdots \oplus I^{2} X^{-2} \oplus I X^{-1} \oplus S \oplus I X \oplus I X^{2} \oplus \cdots
$$

is a nonstrongly graded ring for which $\left\{n \in \mathbb{Z} / R_{n} R_{-n} \subseteq J\left(R_{0}\right)=0\right\}$ is empty.

We do not think that much more information about the group $G$ can be obtained in the general situation described in Proposition 3. However, for the strongly graded case, we can give the following, which is one of the two main results of the paper.

Theorem 5. Let $R=\bigoplus_{\sigma \in G} R_{\sigma}$ be a left perfect strongly G-graded ring such that $R_{1} / J\left(R_{1}\right)$ is a finite direct product of finite-dimensional simple algebras over algebraically closed fields. Then the group $G$ does not contain any infinite abelian subgroup. 
Remark 6. (i) The foregoing result can appear with various disguises. Let us mention three other forms:

(a) Under the same conditions of the theorem, if the group $G$ is abelian then it is finite.

(b) Under the same conditions of the theorem, the group $G$ must either be finite or have an infinite subgroup containing no infinite abelian subgroups.

(c) Under the same conditions of the theorem, all the abelian normal subgroups of $G$ are finite.

Indeed, if the theorem holds then (b) is true and (b) implies (a). But if (a) holds and $G$ is any group grading a strongly graded left perfect ring as in the theorem, then Lemma 1 applied to any abelian subgroup $H$ of $G$ yields via (a) that $H$ is finite. On the other hand, the theorem implies (c) and if (c) holds, then for any group $G$ as in the theorem and any abelian subgroup $H$ of $G$ we take the normalizer $N=N_{G}(H)$ and apply (c) to $R^{(N)}$, which is left perfect by Lemma 1 . We get then that $H$ must be finite.

(ii) Under the assumptions of the theorem, if $G$ is locally finite then $G$ must be finite, due to the well-known fact that any infinite locally finite group contains an infinite abelian subgroup.

The above fact allows us to deal with any of the different equivalent versions of the theorem. So, in particular, our goal is to prove version (a) of the remark by way of contradiction.

The proof of Theorem 5 requires several steps of reduction of the problem.

Lemma 7. If $R$ is a left perfect strongly G-graded ring then $R$ is a crossed product.

Proof. See [10, Corollary I.3.26].

If now $R=A * G$, then $A$ must be left perfect (Lemma 1) and, by factoring by the graded Jacobson radical, we get an also perfect crossed product $A / J(A)$ * $G$. It is then not restrictive to assume that $R=A * G$, where $A$ is semisimple Artinian.

Lemma 8. Let $A * G$ be a crossed product, where $A=A_{1} \times \cdots \times A_{r}$ is a finite direct product of the simple rings $A_{i}$. There exists a (normal) subgroup $H$ of $G$ with finite index such that $A * H \simeq\left(A_{1} * H\right) \times \cdots \times\left(A_{r} * H\right)$, for certain crossed products $A_{i} * H \quad(i=1, \ldots, r)$.

Proof. Since the action of $G$ on $A$ permutes the simple components $A_{i}$ 's, we can define in this way a group homomorphism $G \rightarrow S_{r}$ whose kernel is $H=\left\{\sigma \in G / A_{i}^{\sigma}=A_{i}\right.$ for $\left.i=1, \ldots, r\right\}$. So $H \triangleleft G$ is a subgroup of finite index. But now there is a restricted action of $H$ on each $A_{i}$ and, by considering the restricted twisting $H \times H \hookrightarrow G \times G \stackrel{t}{\rightarrow} U(A) \stackrel{p_{i}}{\rightarrow} U\left(A_{i}\right)$ where $p_{i}$ is the canonical projection, we have crossed products $A_{i} * H(i=1, \ldots, r)$. It is straightforward to see that $A * H \simeq\left(A_{1} * H\right) \times \cdots \times\left(A_{r} * H\right)$.

The foregoing lemma allows us to concentrate on the case where the ground ring $A$ is a finite-dimensional simple algebra over an algebraically closed field, say $K$. This restriction is not needed in the following lemma. In it and the sequel, $\bar{B}$ will denote $B / J(B)$ for any ring $B$. 
Lemma 9. Let $A * G$ be a left perfect crossed product and $H \triangleleft G$ a normal subgroup of finite index. Then $l(\overline{A * H}) \leq l(\overline{A * G})$, where $l(\ldots)$ means composition length.

Proof. Since $H \triangleleft G$, we can consider in $A * G$ the structure of crossed product induced by the action of $G / H$ on $A * H$ so that $A * G=(A * H) * G / H$, and it is well known (see, e.g., ([4], Corollary 4.2 and Theorem 4.3)) that $J(A * H) *$ $G / H \subseteq J(A * G)$ and both intersect $A * H$ in $J(A * H)$. Then we can put $B=\overline{A * H}, G^{\prime}=G / H$, and all we have to prove is that if $B$ is a semisimple Artinian and $G^{\prime}$ is a finite group then $l(B) \leq l\left(\overline{B * G^{\prime}}\right)$. But this is very easy, because the fact that $B \cap J\left(B * G^{\prime}\right)=0$ gives a canonical embedding of $B$ into $\overline{B * G^{\prime}}$ and since $B$ is semisimple Artinian, it is a split monomorphism of left and right $B$-modules, and Proposition 2.1 of [8] tells us that $l(B) \leq l\left(\overline{B * G^{\prime}}\right)$.

A last result is needed before tackling directly the proof of Theorem 5 .

Lemma 10. If $A * G$ is a crossed product where $A$ is a finite-dimensional central simple algebra over the field $K$, then the induced action of $G / G_{\mathrm{inn}}$ on $\overline{A * G_{\mathrm{inn}}}$ is outer.

Proof. As mentioned in the introduction, the action of $\hat{\sigma} \in G / G_{\text {inn }}$ on $A * G_{\text {inn }}$ is the action by conjugation of some representative $\tau$ of $\hat{\sigma}$. If this action is inner in $\overline{A * G_{\text {inn }}}$ then we can consider the inclusion $A \longrightarrow \overline{A * G_{\text {inn }}}$ (see, e.g., [12, Lemma 22.1]) and, since $Z(A)$ is obviously contained in the center of $\overline{A * G_{\text {inn }}}$, we can assert that the action of $\tau$ on $A$ induces the identity map in $K=Z(A)$. By the Skolem-Noether theorem, $\tau \in G_{\text {inn }}$ and so $\hat{\sigma}=\hat{1}$.

Proof of Theorem 5. As already noted, we shall establish version (a) of Remark 6. Then suppose by way of contradiction, that there exists some left perfect crossed product $A * G$ with $G$ infinite abelian. By Proposition 3, $G$ must be a torsion group. As indicated after Lemma 8, we can assume that $A$ is a finite-dimensional simple algebra over the algebraically closed field $K$. Let us consider then the class $\mathscr{H}_{K, G}$ consisting of all the left perfect crossed products $C * H$, where $C$ is a finite-dimensional simple algebra over $K$ and $H \simeq H_{2} / H_{1}$ for some $H_{1} \leq H_{2} \leq G$ with $\left|H_{1}\right|$ and $\left[G: H_{2}\right]$ finite. In $\mathscr{H}_{K, G}$ there must be a crossed product $B * G^{\prime}$ of minimal composition length modulo its Jacobson radical. We shall see that it is not restrictive to assume that $B * G^{\prime}$ is an outer crossed product and hence (see, e.g., [7, Proposition 4.2]) simple and also Artinian because $B * G^{\prime} \in \mathscr{H}_{K, G}$. If it is not outer, we can view $B * G^{\prime}$ as a $G^{\prime} / G_{\text {inn }}^{\prime}$-crossed product over the ground ring $B * G_{\text {inn }}^{\prime}$. By Lemma 1 , this ground ring is left perfect and, on the other hand, it is isomorphic to $B \otimes_{K} K^{t}\left[G_{\text {inn }}^{\prime}\right]$ (see, e.g., [12, Proposition 12.4]). By results of Krempa and Orniński (see [9, Theorem 1.4]), it implies that $K^{t}\left[G_{\text {inn }}^{\prime}\right]$ is left perfect and Theorem 2.6 of [13] asserts that $G_{\text {inn }}^{\prime}$ must be finite. But then $\overline{B * G_{\text {inn }}^{\prime}}$ is a finite-dimensional semisimple Artinian algebra over $K$ (because $K$ is algebraically closed!). By proceeding exactly as in the proof of Lemma 8, we can choose a finite index subgroup $H$ of $G^{\prime}$ containing $G_{\text {inn }}$ so that

$$
\left(\overline{B * G_{\mathrm{inn}}}\right) * H / G_{\mathrm{inn}}^{\prime} \simeq\left(B_{1} * H / G_{\mathrm{inn}}^{\prime}\right) \times \cdots \times\left(B_{r} * H / G_{\mathrm{inn}}^{\prime}\right) .
$$

Now we have

$$
\overline{l\left(\bar{B}_{i} * H / G_{\mathrm{inn}}^{\prime}\right)} \leq l * \overline{\left[\left(\overline{B^{*} G_{\mathrm{inn}}}\right) * H / G_{\mathrm{inn}}^{\prime}\right]},
$$


for $i=1, \ldots, r$, and equality holds if and only if $1=i=r$, i.e., iff $\overline{B * G_{\text {inn }}^{\prime}}$ is simple Artinian (here we have used implicitly the well-known fact that $J\left(A_{1} \times \cdots \times A_{r}\right)=J\left(A_{1}\right) \times \cdots \times J\left(A_{r}\right)$ for a finite direct product of rings). Moreover

$$
\overline{l\left[\left(\overline{B * G_{\mathrm{inn}}^{\prime}}\right) * H / G_{\mathrm{inn}}^{\prime}\right]} \leq \overline{l\left[\overline{B * G_{\mathrm{inn}}^{\prime}} * G^{\prime} / G_{\mathrm{inn}}^{\prime}\right]},
$$

by Lemma 9. But $\left(\overline{B * G_{\mathrm{inn}}^{\prime}}\right) * G^{\prime} / G_{\mathrm{inn}}^{\prime}$ is clearly a factor ring of $B * G^{\prime}$ so that $\overline{\overline{B * G_{\mathrm{inn}}^{\prime}} * G^{\prime} / G_{\mathrm{inn}}^{\prime}}$ is a factor ring (and hence has left composition length smaller or equal than that) of $\overline{B * G^{\prime}}$. But the choice of $B * G^{\prime}$ implies that $l\left(\overline{B_{i} * H / G_{\mathrm{inn}}^{\prime}}\right)$ cannot be strictly smaller than $l\left(\overline{B * G^{\prime}}\right)$ and thereby all the above inequalities are actually equalities. As already noted, this implies that $\overline{B * G_{\text {inn }}^{\prime}}$ is simple Artinian and Lemma 10 ensures that $G^{\prime} / G_{\text {inn }}^{\prime}$ acts outerly on $\overline{B * G_{\text {inn }}^{\prime}}$. Therefore, by replacing $B$ by $\overline{B * G_{\text {inn }}^{\prime}}$ and $G^{\prime}$ by $G^{\prime} / G_{\text {inn }}^{\prime}$ if necessary, we can assume that $B * G^{\prime}$ is an outer simple Artinian crossed product. By the hypothesis, $B=M_{n}(K)$ for a fixed $n \geq 1$. If we restrict the action of $G$ on $B$ to $K=Z(B)$, then we get for each $\sigma \in G$ an automorphism $\sigma_{K}$ of $K$. If now we extend $\sigma_{K}$ in the obvious sense to $B=M_{n}(K)$, i.e., by defining $\hat{\sigma}_{K}\left[\left(x_{i j}\right)\right]=\left(\hat{\sigma}_{K}\left(x_{i j}\right)\right)$, then we get a new automorphism of $B$ that induces in $K$ the same automorphism as $\sigma$. By the Skolem-Noether theorem, $\hat{\sigma}_{k}^{-1} \sigma$ must be an inner automorphism and, consequently, there exists a $P_{\sigma} \in \mathrm{GL}_{n}(K)=U(B)$ so that $P_{\sigma} b^{\sigma} P_{\sigma}^{-1}=\hat{\sigma}_{k}(b)$ for all $b \in B$. If in the crossed product $B * G^{\prime}$ we replace $\bar{\sigma}$ by $P_{\sigma} \bar{\sigma}$, then we get an equivalent crossed product structure but with the nice property that the new action

$$
\begin{aligned}
& G^{\prime} \stackrel{\varphi^{\prime}}{\rightarrow} \operatorname{Aut}(B) \\
& \sigma \stackrel{\hat{\sigma}_{k}}{ }
\end{aligned}
$$

is a group homomorphism and the associated twisting $t^{\prime}$ satisfies the property that $b^{\sigma \tau}=t^{\prime}(\sigma, \tau) b^{\sigma \tau} t^{\prime}(\sigma, \tau)^{-1}$ for any $\sigma, \tau \in G^{\prime}$ and every $b \in B$. In other words, $t^{\prime}(\sigma, \tau) \in Z(B)=K$ for every $\sigma, \tau \in G^{\prime}$. It can be seen in a straightforward way that, with the new structure, $B * G^{\prime} \simeq M_{n}\left(K * G^{\prime}\right)$ for the $G^{\prime}$-crossed product over $K$ defined by

$$
\begin{gathered}
G^{\prime} \stackrel{\varphi^{\prime}}{\longrightarrow} \operatorname{Aut}(B) \longrightarrow \operatorname{Aut}(K), \\
G^{\prime} \times G^{\prime} \longrightarrow U(K) \hookrightarrow \mathrm{GL}_{n}(K)=U(B) .
\end{gathered}
$$

Now $K * G^{\prime} \in \mathscr{H}_{K, G}$ and $l\left(K * G^{\prime}\right)$ must equal $l\left(B * G^{\prime}\right)=l\left(M_{n}\left(K * G^{\prime}\right)\right)$ by the choice of $B * G^{\prime}$. Therefore $n=1$ so that $B=K$ is a field. We claim now that $G^{\prime}$ cannot have nontrivial finite subgroups, and this would be a contradiction because the group $G^{\prime}$ is infinite torsion abelian. Suppose $H^{\prime}<G^{\prime}$ is a finite subgroup. Then we can give $K * G^{\prime}$ a structure of $G^{\prime} / H^{\prime}$-crossed product over $K * H^{\prime}$, which is a finite-dimensional simple algebra over $K\left(K * G^{\prime}\right.$ and hence $K * H^{\prime}$ are outer!) and, consequently, $\left(K * H^{\prime}\right) * G / H^{\prime} \simeq M_{p}\left(K * G^{\prime} / H^{\prime}\right)$ so that $K * G^{\prime} / H^{\prime}$ is a simple Artinian ring with composition length $\leq l\left(K * G^{\prime}\right)$. By minimality both must be equal, implying that $p=1$. Thus $K * H^{\prime} \simeq K$ and thereby $H^{\prime}=1$.

Remark 11. The proof of Theorem 1.5 ultimately leans upon the result of Reid stating that if $K^{t}[G]$ is a twisted group algebra that is left perfect then $G$ cannot contain an infinite abelian subgroup [13, Theorem 2.6]. If instead of 
left perfectness we consider that the ring $R=\bigoplus_{\sigma \in G} R_{\sigma}$ is left Artinian, then we can lean upon the somehow more general result of Passman [11, Theorem 5.4] and reconstruct almost exactly the above proof to show

Theorem 12. Let $R=\bigoplus_{\sigma \in G} R_{\sigma}$ be a left Artinian strongly graded ring such that $R_{1} / J\left(R_{1}\right)$ is a finite direct product of finite-dimensional simple algebras over nondenumerable algebraically closed fields. Then the group $G$ is finite.

\section{REFERENCES}

1. F. W. Anderson and U. R. Fuller, Rings and categories of modules, Springer-Verlag, New York, 1974.

2. H. Bass, Finitistic dimension and a homological generalization of semiprimary rings, Trans. Amer. Math. Soc. 95 (1960), 466-488.

3. V. Camilo and K. Fuller, On graded rings with finiteness conditions, Proc. Amer. Math. Soc. 86 (1982), 1-6.

4. M. Cohen and S. Montgomery, Group-graded rings, smash products and group actions, Trans. Amer. Math. Soc. 282 (1984), 237-258.

5. _ Addendum to Group-graded rings, smash products and group actions, Trans. Amer. Math. Soc. 300 (1987), 810-811.

6. A. Jensen and S. Jöndrup, Smash products, group actions and group graded rings, preprint.

7. G. Karpilovsky, The algebraic structure of crossed products, Math. Studies., vol. 142, North Holland, 1987.

8. J. Keok Park, Artinian skew group rings, Proc. Amer. Math. Soc. 75 (1979), 1-7.

9. J. Krempa and J. Orniński, Semilocal, semiperfect and perfect tensor products, Bull. Acad. Polon. Sci. XXVIII (1980), 249-256.

10. C. Năstăstescu and F. Van Oystaeyen, Graded ring theory, Math. Library, vol. 28, North Holland, 1980.

11. D. S. Passman, Radicals of twisted group rings II. Proc. London Math. Soc. (3) 22 (1971), 633-51.

12. _ Infinite crossed products, Academic Press, 1989.

13. A. Reid, Twisted-group algebras which are Artinian, perfect or self-injective Bull. London Math. Soc. 7 (1975), 166-170.

14. G. Renault, Sur les anneaux de groupe, C. R. Acad. Sci. Paris. Ser. A-B 273 (1971).

15. S. M. Woods, On perfect group rings, Proc. Amer. Math. Soc. 27 (1971), 49-52.

Departamento de Matemáticas, Universidad de Murcia, 30100 Espinardo, MuRCIA, SPAIN

E-mail address: MSAORINC@FC.UM.ES 